\title{
Research Article \\ Patient's Compliance with Oral Antibiotics Treatments at Community Health Centers in Surabaya: A 20-KAO Questionnaire Development
}

\author{
Ilil Maidatuz Zulfa ${ }^{*}$ \\ Widya Handayani 2 \\ ${ }^{1}$ Department of Community Pharmacy, \\ Akademi Farmasi Surabaya, Surabaya, \\ East Java, Indonesia \\ 2Department of Clinical Pharmacy, \\ Akademi Farmasi Surabaya, Surabaya, \\ East Java, Indonesia \\ *email: ililmaidatuz@gmail.com \\ Keywords: \\ Antibiotics \\ Compliance \\ Community Health Centre \\ Questionnaire
}

\begin{abstract}
The irrational and incompliance use of antibiotics has been correlated to bacterial resistance. Several methods evaluated patients' compliance with oral antibiotics have been conducted. However, a standard questionnaire for evaluating oral antibiotics compliance in Indonesian has not been developed yet. This study was conducted to record the content validity of the developed questionnaire called 20-KAO to assess compliance with oral antibiotics. The validity content test was conducted through six experts review using the Item Content Validity Index (I-CVI) and Scale-Content Validity Index (S-CVI). The experts were also requested to provide recommendations for each item, whether revisions or deletion. After the review process, the number of questions remains unchanged. A total of 19 out of 20 items had an ICVI of 1.00, and S-CVI was calculated at 0.98 . Therefore, 20 items of the 20-KAO questionnaire have excellent content validity. However, future construct validity and reliability test to analyze the responses of targeted respondents and the questionnaire's consistency are needed.
\end{abstract}

Received: September $11^{\text {th }}, 2020$

Accepted: October 30th, 2020

Published: November 30th, 2020

(C) 2020 Ilil Maidatuz Zulfa, Widya Handayani. Published by Institute for Research and Community Services Universitas Muhammadiyah Palangkaraya. This is an Open Access article under the CC-BY-SA License (http://creativecommons.org/licenses/by-sa/4.0/). DOI: https://doi.org/10.33084/bjop.v3i4.1660

\section{INTRODUCTION}

Antibiotics are chemical substances produced by microorganisms that can inhibit or kill bacteria (Fair \& Tor, 2014). Antibiotics usage for an infection in the low and middle-income countries has increased up to $65 \%$ from 2010-2015 (Klein et al., 2018). An increase has followed the increase in usage in bacterial resistance. The irrational use of antibiotics, such as treating viral infections and usage on the farm and fisheries, correlates with bacterial resistance (Economou \& Gousia, 2015). Furthermore, the patient's compliance in taking their oral antibiotics also contributed to bacterial resistance prevalence (Moradi et al., 2013).

Several studies evaluated the patient's compliance with oral antibiotics have been conducted using various methods. Measuring compliance using objective or subjective methods has its particular limitations (Jin et al., 2008). Examples of objective methods in assessing patient compliance are pill count and Medication Event Monitoring System (MEMS). Pill count is a physical count of the number of pills that remain and compares to the prescription's instruction (Lam \& Fresco, 2015). The MEMS is a medication container contains a microelectronic chip that records the date and time of opening of every bottle (Llor et al., 2013). Although MEMS measures compliance accurately, it cannot be used in an everyday situation. Besides, pill count cannot describe specifically the aspects of compliance, such as timing, dosing, and taking compliance (Willams et al., 2013). Hence, a subjective method such as using a questionnaire is preferable because of less cost, nonjudgmental, non-threatening, quick, and comfortable in 
collecting results (Lam \& Fresco, 2015; Llor et al., 2013;

Ponto, 2015).

Various questionnaires such as Medication Adherence Questionnaire (MAQ), Medication Adherence Rating Scale (MARS), Patient Medication Adherence Questionnaire (PMAQ), Morisky Medication Adherence Scale (MMAS), etc. have been developed and validated to measure compliance (Morisky et al., 1986; Thompson et al., 2000; Duong et al., 2001). However, the questionnaires have not been translated and validated into Bahasa Indonesia for measuring compliance with oral antibiotics treatment in Indonesia. A study has been conducted to validate the English version of Morisky 3, 4, and 5-item questionnaires in assessing oral antibiotics compliance (Treibich \& Ventelou, 2017).

In Indonesia, measuring oral antibiotics compliance usually uses pill count. Therefore, there has been no questionnaire developed yet. Hence, developing a standard questionnaire for evaluating oral antibiotics compliance in Bahasa Indonesia is essentials. In developing a questionnaire, validity tests are needed in order to evaluate whether it measures what it is supposed to measure or not (Setia, 2017). Validity tests are classified into two broad categories, viz internal and external validity (Bolarinwa, 2015). Internal validity includes content validity that measures the degree to which the instrument comprehensively assesses the construct of interest usually conducted before external validity. Therefore, this study was conducted to record the developed questionnaire's content validity assessing compliance with oral antibiotics.

\section{MATERIALS AND METHODS}

This study was conducted observationally from August to September 2020 to establish the evidence of content validity of the developed questionnaire, namely 20-KAO from experts.

\section{0-KAO Questionnaire}

The 20-KAO questionnaire was developed in Bahasa Indonesia and aimed to assess short-course oral antibiotics usage compliance. The name 20-KAO was developed from 20 items in assessing compliance with oral antibiotics or in Bahasa Indonesia: Kepatuhan Antibiotik Oral (KAO). The questionnaire was developed in four sections and contained 20 questions. Section one contained ten questions to evaluate whether the patients understood the dosage regimens and took their dosage correctly or not and the reasons behind their action in taking the dosage. Section two rated the patient's understanding, compliance, and the reasons in how many times they took the dosage daily through five questions. In the third section, it was assessed how patients gave an interval between their dosages and the reasons why they did it. In the last section, the patient's understanding of the duration of taking the antibiotics, and whether they had stopped taking the antibiotics before it should be stopped, and their reasons to do so. Additionally, patients were also asked to fill in the number of pills left in the questionnaire and if they used other nonprescribed medication.

\section{Content validation}

The literature shows that the ideal number of content experts needed in a validation study is still controversial. However, the suggestions are between three and ten experts (Polit \& Beck, 2006). In this study, a total of six experts were selected. Three of them were academic experts, while three remained were registered pharmacists. These experts' proportion were designed intentionally so that the review results would reflect academic and practical opinions. The academic experts were selected based on their experiences in developing a questionnaire and the pharmacists' experts' experiences in giving patient consultation to improve the patient's compliance. The experts were invited from educational 
institutions and community health centers in Surabaya (they were registered pharmacists in Wonokromo, Gayungan, and Kalirungkut Community Health Centers and academic lecturers in clinical as well as community pharmacy at Universitas Airlangga and Akademi Farmasi Surabaya). After getting their approval, the researcher sent an informed consent form, an information cover letter, and the questionnaire attached to the evaluation criteria. The evaluation form contained an explanation of the validation procedure. The experts were asked to assess the relevance of each question in the questionnaire.

The relevance meant whether all the items in the 20-KAO questionnaire referred to measure the antibiotics usage compliance in dose, frequency, and the duration of therapy. To determine the relevance of each item, a four scale was used ( 1 = not relevant, 2 = somewhat relevant, $3=$ quite relevant, $4=$ highly relevant). The experts were also requested to provide recommendations for each item, either revisions or deletion. The maximum time for validating the questionnaire for each expert was two weeks, and they were requested to return the result through email or in-person to the researcher. The response from the experts was analyzed through Content Validity Index (CVI), specifically Item Content Validity Index (I-CVI) and Scale-Content Validity Index (S-CVI). The I-CVI was calculated in every item from the number of experts giving 3 or 4 scores divided by the total number of experts, while S-CVI was computed as the average of I-CVI from all the items. The questionnaire would qualify to be content valid if theS-CVI greater than 0.90 .

\section{RESULTS AND DISCUSSION}

All of the six experts invited were agreed to participate. They were registered pharmacists in Wonokromo, Gayungan, and Kalirungkut Community Health
Centers and academic lecturers in clinical and community pharmacy at Universitas Airlangga and Akademi Farmasi Surabaya. All experts are deliberately selected from Surabaya to facilitate communication. The mean age of the experts was $41.5(\mathrm{SD}=9.16)$ years. After the validation process, the number of question items in the 20-KAO questionnaire remained unchanged. There were 19 out of 20 items that had an I-CVI of 1.00. Therefore, S-CVI was calculated at 0.98 as shown in Table I.

Table I. Result for the content validation of 20-KAO questionnaire

\begin{tabular}{|c|c|c|c|c|c|c|c|c|c|}
\hline \multirow{2}{*}{ Section } & \multirow{2}{*}{$\begin{array}{c}\text { Item } \\
\text { Number }\end{array}$} & \multirow{2}{*}{$\begin{array}{l}\text { Question } \\
\text { description }\end{array}$} & \multicolumn{6}{|c|}{ Expert Raters } & \multirow{2}{*}{$\begin{array}{c}\text { I- } \\
\text { CVI }\end{array}$} \\
\hline & & & 1 & 2 & 3 & 4 & 5 & 6 & \\
\hline \multirow[t]{10}{*}{$\begin{array}{c}\text { Section } \\
\text { I }\end{array}$} & Q1 & $\begin{array}{l}\text { Knowledge } \\
\text { of } \\
\text { prescribed } \\
\text { antibiotic } \\
\text { dose at one } \\
\text { time }\end{array}$ & 3 & 4 & 4 & 3 & 4 & 3 & 1.00 \\
\hline & Q2 & $\begin{array}{l}\text { Experience } \\
\text { in taking a } \\
\text { higher } \\
\text { dosage at } \\
\text { one time }\end{array}$ & 4 & 3 & 4 & 3 & 4 & 3 & 1.00 \\
\hline & Q3 & $\begin{array}{l}\text { Reasons for } \\
\text { Q2 Answer }\end{array}$ & 4 & 3 & 4 & 3 & 4 & 3 & 1.00 \\
\hline & Q4 & $\begin{array}{l}\text { Experience } \\
\text { in taking a } \\
\text { lower } \\
\text { dosage at } \\
\text { one time }\end{array}$ & 4 & 3 & 4 & 3 & 4 & 3 & 1.00 \\
\hline & Q5 & $\begin{array}{l}\text { Reasons fo } \\
\text { Q4 Answer }\end{array}$ & 4 & 3 & 4 & 3 & 4 & 3 & 1.00 \\
\hline & Q6 & $\begin{array}{l}\text { Knowledge } \\
\text { of } \\
\text { prescribed } \\
\text { antibiotic } \\
\text { dose daily }\end{array}$ & 3 & 4 & 4 & 4 & 4 & 3 & 1.00 \\
\hline & Q7 & $\begin{array}{l}\text { Experience } \\
\text { in taking } \\
\text { higher } \\
\text { dosage } \\
\text { daily }\end{array}$ & 4 & 4 & 4 & 3 & 4 & 3 & 1.00 \\
\hline & Q8 & $\begin{array}{l}\text { Reasons for } \\
\text { Q7 Answer }\end{array}$ & 4 & 4 & 4 & 3 & 4 & 3 & 1.00 \\
\hline & Q9 & $\begin{array}{l}\text { Experience } \\
\text { in taking } \\
\text { lower } \\
\text { dosage } \\
\text { daily }\end{array}$ & 4 & 4 & 4 & 3 & 4 & 3 & 1.00 \\
\hline & Q10 & $\begin{array}{l}\text { Reasons for } \\
\text { Q9 Answer }\end{array}$ & 4 & 4 & 4 & 3 & 4 & 3 & 1.00 \\
\hline \multirow[t]{2}{*}{$\begin{array}{c}\text { Section } \\
\text { II }\end{array}$} & Q11 & $\begin{array}{l}\text { Knowledge } \\
\text { of times } \\
\text { taken } \\
\text { prescribed } \\
\text { antibiotics } \\
\text { daily }\end{array}$ & 4 & 4 & 4 & 2 & 4 & 2 & 0.67 \\
\hline & Q12 & $\begin{array}{l}\text { Experience } \\
\text { in taking } \\
\text { more } \\
\text { frequent }\end{array}$ & 4 & 3 & 4 & 3 & 4 & 3 & 1.00 \\
\hline
\end{tabular}




\begin{tabular}{|c|c|c|c|c|c|c|c|c|c|}
\hline & Q13 & $\begin{array}{l}\text { Reasons for } \\
\text { Q12 } \\
\text { Answer }\end{array}$ & 4 & 3 & 4 & 3 & 4 & 3 & 1.00 \\
\hline & Q14 & $\begin{array}{l}\text { Experience } \\
\text { in taking } \\
\text { less } \\
\text { frequent }\end{array}$ & 3 & 3 & 4 & 3 & 4 & 3 & 1.00 \\
\hline & Q15 & $\begin{array}{l}\text { Reasons for } \\
\text { Q14 } \\
\text { Answer }\end{array}$ & 3 & 3 & 4 & 3 & 4 & 3 & 1.00 \\
\hline $\begin{array}{c}\text { Section } \\
\text { III }\end{array}$ & Q16 & $\begin{array}{l}\text { The time } \\
\text { interval } \\
\text { between } \\
\text { dose }\end{array}$ & 3 & 3 & 4 & 4 & 4 & 3 & 1.00 \\
\hline \multirow[t]{5}{*}{$\begin{array}{c}\text { Section } \\
\text { IV }\end{array}$} & Q17 & $\begin{array}{l}\text { Knowledge } \\
\text { of } \\
\text { prescribed } \\
\text { antibiotics } \\
\text { duration }\end{array}$ & 3 & 4 & 4 & 4 & 4 & 3 & 1.00 \\
\hline & Q18 & $\begin{array}{l}\text { Whether } \\
\text { has stopped } \\
\text { their } \\
\text { antibiotics } \\
\text { course }\end{array}$ & 4 & 3 & 4 & 4 & 4 & 3 & 1.00 \\
\hline & Q19 & $\begin{array}{l}\text { Reasons for } \\
\text { Q19 } \\
\text { Answer }\end{array}$ & 4 & 3 & 4 & 4 & 4 & 3 & 1.00 \\
\hline & Q20 & $\begin{array}{l}\text { The } \\
\text { number of } \\
\text { pills } \\
\text { remaining }\end{array}$ & 4 & 4 & 4 & 4 & 4 & 3 & 1.00 \\
\hline & \multicolumn{2}{|c|}{ Total agreement $=0.95^{\mathrm{a}}$} & \multicolumn{7}{|c|}{$\mathrm{S}-\mathrm{CVI}=0.98$} \\
\hline
\end{tabular}

aNumber of items that achieved the I-CVI of 1.00 divided by the total number of items to be validated in the questionnaire

Table I showed that the questionnaire had excellent content validity in measuring oral antibiotic compliance. However, the experts gave some editorial revisions to make the sentences more comfortable to be understood, as presented in Table II.

Table II. Editorial revision of 20-KAO questionnaire

\begin{tabular}{|c|c|c|c|c|}
\hline Item & $\begin{array}{l}\text { Question } \\
\text { description }\end{array}$ & $\begin{array}{c}\text { Original } \\
\text { Question } \\
\text { Sentences } \\
\text { (Bahasa } \\
\text { Indonesia) }\end{array}$ & $\begin{array}{c}\text { After } \\
\text { Editorial } \\
\text { Revision } \\
\text { (Bahasa } \\
\text { Indonesia) } \\
\end{array}$ & $\begin{array}{c}\text { After } \\
\text { Editorial } \\
\text { Revision } \\
\text { (English) }\end{array}$ \\
\hline Q1 & $\begin{array}{l}\text { Knowledge } \\
\text { of } \\
\text { prescribed } \\
\text { antibiotic } \\
\text { dose at one } \\
\text { time }\end{array}$ & $\begin{array}{l}\text { Berapa } \\
\text { tablet/kapsul } \\
\text { antibiotik dari } \\
\text { puskesmas } \\
\text { yang } \\
\text { seharusnya } \\
\text { anda konsumsi } \\
\text { tiap kali } \\
\text { minum? }\end{array}$ & $\begin{array}{l}\text { Berapa jumlah } \\
\text { tablet/kapsul } \\
\text { antibiotik yang } \\
\text { seharusnya } \\
\text { anda konsumsi } \\
\text { tiap kali } \\
\text { minum? }\end{array}$ & $\begin{array}{l}\text { How many } \\
\text { pills of } \\
\text { antibiotic } \\
\text { should you } \\
\text { take at one } \\
\text { time? }\end{array}$ \\
\hline Q2 & $\begin{array}{l}\text { Experience } \\
\text { in taking } \\
\text { higher } \\
\text { dosage at } \\
\text { one time }\end{array}$ & $\begin{array}{l}\text { Apakah Anda } \\
\text { pernah } \\
\text { mengkonsumsi } \\
\text { lebih dari } \\
\text { jumlah } \\
\text { tersebut tiap } \\
\text { kali minum? }\end{array}$ & $\begin{array}{l}\text { Apakah Anda } \\
\text { pernah } \\
\text { mengkonsumsi } \\
\text { obat antibiotik } \\
\text { lebih dari } \\
\text { jumlah } \\
\text { tersebut tiap } \\
\text { kaliminum? }\end{array}$ & $\begin{array}{l}\text { Have you } \\
\text { taken more } \\
\text { antibiotic } \\
\text { pills than } \\
\text { that at one } \\
\text { time? }\end{array}$ \\
\hline Q3 & $\begin{array}{l}\text { Reasons for } \\
\text { Q2 Answer }\end{array}$ & $\begin{array}{l}\text { Apa alasan } \\
\text { Anda? }\end{array}$ & - & $\begin{array}{l}\text { Please } \\
\text { explain } \\
\text { your } \\
\text { reasons. }\end{array}$ \\
\hline Q4 & $\begin{array}{l}\text { Experience } \\
\text { in taking }\end{array}$ & $\begin{array}{l}\text { Apakah Anda } \\
\text { pernah } \\
\text { mengkonsumsi }\end{array}$ & $\begin{array}{l}\text { Apakah Anda } \\
\text { pernah } \\
\text { mengkonsumsi }\end{array}$ & $\begin{array}{ll}\text { Have } & \text { you } \\
\text { taken } & \text { less }\end{array}$ \\
\hline
\end{tabular}

\begin{tabular}{|c|c|c|c|c|}
\hline & $\begin{array}{l}\text { lower } \\
\text { dosage at } \\
\text { one time }\end{array}$ & $\begin{array}{l}\text { kurang dari } \\
\text { jumlah } \\
\text { tersebut tiap } \\
\text { kaliminum? }\end{array}$ & $\begin{array}{l}\text { obat antibiotik } \\
\text { kurang dari } \\
\text { jumlah } \\
\text { tersebut tiap } \\
\text { kali minum? }\end{array}$ & $\begin{array}{l}\text { antibiotic } \\
\text { pills than } \\
\text { that at one } \\
\text { time? }\end{array}$ \\
\hline Q5 & $\begin{array}{l}\text { Reasons for } \\
\text { Q4 Answer }\end{array}$ & $\begin{array}{l}\text { Apa alasan } \\
\text { Anda? }\end{array}$ & - & $\begin{array}{l}\text { Please } \\
\text { explain } \\
\text { your } \\
\text { reasons. }\end{array}$ \\
\hline Q6 & $\begin{array}{l}\text { Knowledge } \\
\text { of } \\
\text { prescribed } \\
\text { antibiotic } \\
\text { dose daily }\end{array}$ & $\begin{array}{l}\text { Berapa } \\
\text { tablet/kapsul } \\
\text { antibiotik dari } \\
\text { puskesmas } \\
\text { yang } \\
\text { seharusnya } \\
\text { anda konsumsi } \\
\text { dalam satu } \\
\text { hari? }\end{array}$ & $\begin{array}{l}\text { Berapa jumlah } \\
\text { tablet/kapsul } \\
\text { antibiotik yang } \\
\text { seharusnya } \\
\text { anda konsumsi } \\
\text { dalam satu } \\
\text { hari? }\end{array}$ & $\begin{array}{l}\text { How many } \\
\text { pills of } \\
\text { antibiotic } \\
\text { should you } \\
\text { take in one } \\
\text { day? }\end{array}$ \\
\hline Q7 & $\begin{array}{l}\text { Experience } \\
\text { in taking } \\
\text { higher } \\
\text { dosage } \\
\text { daily }\end{array}$ & $\begin{array}{l}\text { Apakah Anda } \\
\text { pernah } \\
\text { mengkonsumsi } \\
\text { lebih dari } \\
\text { jumlah } \\
\text { tersebut } \\
\text { dalam satu } \\
\text { hari? }\end{array}$ & $\begin{array}{l}\text { Apakah Anda } \\
\text { pernah } \\
\text { mengkonsumsi } \\
\text { obat antibiotik } \\
\text { lebih dari } \\
\text { jumlah dalam } \\
\text { satu hari? }\end{array}$ & $\begin{array}{l}\text { Have you } \\
\text { taken more } \\
\text { antibiotic } \\
\text { pills than } \\
\text { that in one } \\
\text { day? }\end{array}$ \\
\hline Q8 & $\begin{array}{l}\text { Reasons for } \\
\text { Q7 Answer }\end{array}$ & $\begin{array}{l}\text { Apa alasan } \\
\text { Anda? }\end{array}$ & - & $\begin{array}{l}\text { Please } \\
\text { explain } \\
\text { your } \\
\text { reasons. }\end{array}$ \\
\hline Q9 & $\begin{array}{l}\text { Experience } \\
\text { in taking } \\
\text { lower } \\
\text { dosage } \\
\text { daily }\end{array}$ & $\begin{array}{l}\text { Apakah Anda } \\
\text { pernah } \\
\text { mengkonsumsi } \\
\text { kurang dari } \\
\text { jumlah } \\
\text { tersebut } \\
\text { dalam satu } \\
\text { hari? }\end{array}$ & $\begin{array}{l}\text { Apakah Anda } \\
\text { pernah } \\
\text { mengkonsumsi } \\
\text { obat antibiotik } \\
\text { kurang dari } \\
\text { jumlah } \\
\text { tersebut } \\
\text { dalam satu } \\
\text { hari? }\end{array}$ & $\begin{array}{l}\text { Have you } \\
\text { taken less } \\
\text { antibiotic } \\
\text { pills than } \\
\text { that in one } \\
\text { day? }\end{array}$ \\
\hline Q10 & $\begin{array}{l}\text { Reasons for } \\
\text { Q9 Answer }\end{array}$ & $\begin{array}{l}\text { Apa alasan } \\
\text { Anda? }\end{array}$ & - & $\begin{array}{l}\text { Please } \\
\text { explain } \\
\text { your } \\
\text { reasons. }\end{array}$ \\
\hline Q11 & $\begin{array}{l}\text { Knowledge } \\
\text { of times } \\
\text { taken } \\
\text { prescribed } \\
\text { antibiotics } \\
\text { daily }\end{array}$ & $\begin{array}{l}\text { Berapa kali } \\
\text { seharusnya } \\
\text { Anda minum } \\
\text { antibiotik dari } \\
\text { puskesmas } \\
\text { beberapa hari } \\
\text { yang lalu } \\
\text { dalam satu } \\
\text { hari? }\end{array}$ & $\begin{array}{l}\text { Berapa kali } \\
\text { dalam satu } \\
\text { hari } \\
\text { seharusnya } \\
\text { Anda minum } \\
\text { antibiotik dari } \\
\text { puskesmas } \\
\text { sesuai } \\
\text { peresepan } \\
\text { dokter? }\end{array}$ & $\begin{array}{l}\text { How many } \\
\text { times } \\
\text { should you } \\
\text { take your } \\
\text { antibiotic as } \\
\text { prescribed } \\
\text { in one day? }\end{array}$ \\
\hline Q12 & $\begin{array}{l}\text { Experience } \\
\text { in taking } \\
\text { more } \\
\text { frequent }\end{array}$ & $\begin{array}{l}\text { Apakah Anda } \\
\text { pernah } \\
\text { mengkonsumsi } \\
\text { lebih dari itu } \\
\text { dalam satu } \\
\text { hari? }\end{array}$ & & $\begin{array}{l}\text { Have you } \\
\text { taken more } \\
\text { frequently } \\
\text { than that? }\end{array}$ \\
\hline Q13 & $\begin{array}{l}\text { Reasons for } \\
\text { Q12 } \\
\text { Answer }\end{array}$ & $\begin{array}{l}\text { Apa alasan } \\
\text { Anda? }\end{array}$ & & $\begin{array}{l}\text { Please } \\
\text { explain } \\
\text { your } \\
\text { reasons. }\end{array}$ \\
\hline Q14 & $\begin{array}{l}\text { Experience } \\
\text { in taking } \\
\text { less } \\
\text { frequent }\end{array}$ & $\begin{array}{l}\text { Apakah Anda } \\
\text { pernah } \\
\text { mengkonsumsi } \\
\text { kurang dari } \\
\text { itu dalam } \\
\text { satu hari? }\end{array}$ & & $\begin{array}{l}\text { Have you } \\
\text { taken less } \\
\text { frequently } \\
\text { than that? }\end{array}$ \\
\hline Q15 & $\begin{array}{l}\text { Reasons for } \\
\text { Q14 } \\
\text { Answer }\end{array}$ & $\begin{array}{l}\text { Apa alasan } \\
\text { Anda? }\end{array}$ & & $\begin{array}{l}\text { Please } \\
\text { explain } \\
\text { your } \\
\text { reasons. }\end{array}$ \\
\hline Q16 & $\begin{array}{l}\text { The time } \\
\text { interval } \\
\text { between } \\
\text { dosage }\end{array}$ & $\begin{array}{l}\text { Bagaimana } \\
\text { Anda memberi } \\
\text { jeda waktu } \\
\text { dalam } \\
\text { meminum } \\
\text { antibiotik } \\
\text { Anda? }\end{array}$ & & $\begin{array}{l}\text { How do } \\
\text { you give } \\
\text { interval } \\
\text { between } \\
\text { your } \\
\text { antibiotic } \\
\text { dosage? }\end{array}$ \\
\hline
\end{tabular}




\begin{tabular}{|c|c|c|c|c|}
\hline Q17 & $\begin{array}{l}\text { Knowledge } \\
\text { of } \\
\text { prescribed } \\
\text { antibiotics } \\
\text { duration }\end{array}$ & $\begin{array}{l}\text { Untuk berapa } \\
\text { hari } \\
\text { seharusnya } \\
\text { Anda minum } \\
\text { antibiotik } \\
\text { Anda? }\end{array}$ & $\begin{array}{l}\text { Sejak kapan } \\
\text { Anda minum } \\
\text { antibiotik? }\end{array}$ & $\begin{array}{l}\text { Since when } \\
\text { do you take } \\
\text { your } \\
\text { antibiotics } \\
\text { course? }\end{array}$ \\
\hline Q18 & $\begin{array}{l}\text { Whether } \\
\text { has stopped } \\
\text { their } \\
\text { antibiotics } \\
\text { course }\end{array}$ & $\begin{array}{l}\text { Apakah Anda } \\
\text { telah berhenti } \\
\text { meminum } \\
\text { antibiotik } \\
\text { Anda sebelum } \\
\text { hari ini? }\end{array}$ & & $\begin{array}{l}\text { Have you } \\
\text { stopped } \\
\text { your } \\
\text { antibiotic } \\
\text { course } \\
\text { before } \\
\text { today? }\end{array}$ \\
\hline Q19 & $\begin{array}{l}\text { Reasons for } \\
\text { Q19 } \\
\text { Answer }\end{array}$ & $\begin{array}{l}\text { Apa alasan } \\
\text { Anda? }\end{array}$ & & $\begin{array}{l}\text { Please } \\
\text { explain } \\
\text { your } \\
\text { reasons. }\end{array}$ \\
\hline Q20 & $\begin{array}{l}\text { The number } \\
\text { of pills } \\
\text { remaining }\end{array}$ & $\begin{array}{l}\text { Berapa } \\
\text { antibiotik anda } \\
\text { yang tersisa? }\end{array}$ & $\begin{array}{l}\text { Berapa Jumlah } \\
\text { antibiotik anda } \\
\text { yang tersisa? }\end{array}$ & $\begin{array}{l}\text { How many } \\
\text { pills of } \\
\text { antibiotics } \\
\text { do you } \\
\text { have left? }\end{array}$ \\
\hline
\end{tabular}

Among the 20 items validated, the only item with I-CVI less than 1.00 and significant editorial revision was Q11. The Q11 was developed to assess whether the patients understand how many times they should take their antibiotics or not. Two of the six experts suggested changing the sentence structured because the original sentence was too complicated. Changes in the structure of the questions were then carried out after consultation with these experts. The final form of the 20-KAO questionnaire after the content validity process was shown in Table III.

Table III. The 20-KAO questionnaire

\begin{tabular}{|c|c|c|c|c|c|}
\hline \multicolumn{6}{|c|}{ Identities } \\
\hline \multicolumn{3}{|c|}{$\begin{array}{l}\text { Nama atau Inisial } \\
\text { (Name or Initials) }\end{array}$} & \multicolumn{3}{|c|}{ (n) } \\
\hline \multicolumn{3}{|c|}{$\begin{array}{l}\text { Jenis Kelamin } \\
\text { (Gender) }\end{array}$} & \multicolumn{2}{|c|}{ Perempuan (Female) } & Laki-laki (Male) \\
\hline \multicolumn{3}{|c|}{$\begin{array}{l}\text { Usia dalam tahun } \\
\text { (Age in y.o) }\end{array}$} & \multicolumn{3}{|c|}{ 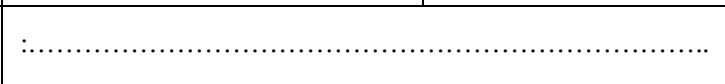 } \\
\hline \multicolumn{3}{|c|}{$\begin{array}{l}\text { Apakah anda memiliki riwayat penyakit ? Jika IYA, harap sebutkan. } \\
\text { (Do you have comorbids? If YES, please mention it) }\end{array}$} & \multicolumn{3}{|c|}{ : } \\
\hline \multicolumn{3}{|c|}{$\begin{array}{l}\text { Apakah anda mengkonsumsi obat lain selain yang diresepkan untuk } \\
\text { anda? Jika IYA, harap sebutkan. } \\
\text { (Do you take any other medications out of prescribed? If YES, please } \\
\text { mention it) }\end{array}$} & \multicolumn{3}{|c|}{ (n. } \\
\hline \multicolumn{6}{|c|}{ 20-KAO Questionnaire } \\
\hline \multirow{4}{*}{$\begin{array}{c}\text { Bagian } 1 \\
(\text { Section 1) }\end{array}$} & $\begin{array}{l}\text { 1. Berapa jumlah tablet/ kapsul } \\
\text { antibiotik yang seharusnya anda } \\
\text { konsumsi tiap kali minum? } \\
\text { (How many pills of antibiotic should } \\
\text { you take at one time?) }\end{array}$ & $\therefore \ldots$ & & & \\
\hline & $\begin{array}{l}\text { 2. Apakah Anda pernah } \\
\text { mengkonsumsi obat antibiotik } \\
\text { lebih dari jumlah tersebut tiap kali } \\
\underline{\text { minum? }} \\
\text { (Have you taken more antibiotic pills } \\
\text { than that at one time?) }\end{array}$ & $\begin{array}{l}: \text { Ya }(\text { Yes }) \\
\square\end{array}$ & $\begin{array}{c}: \text { Tidak (No) } \\
\square\end{array}$ & $\begin{array}{l}\text { 3. Apa A } \\
\text { (Pleas }\end{array}$ & ? \\
\hline & $\begin{array}{l}\text { 4. } \begin{array}{l}\text { Apakah Anda } \\
\text { mengkonsumsi obat antibiotik } \\
\text { kurang dari jumlah tersebut tiap kali }\end{array} \\
\text { minum? } \\
\text { (Have you taken less antibiotic pills } \\
\text { than that at one time?) }\end{array}$ & $\begin{array}{l}: \text { Ya (Yes) } \\
\square\end{array}$ & : Tidak (No) & $\begin{array}{l}\text { 5. Apa A } \\
\text { (Pleas }\end{array}$ & ? \\
\hline & $\begin{array}{l}\text { 6. Berapa jumlah tablet/ kapsul } \\
\text { antibiotik yang seharusnya anda } \\
\text { konsumsi dalam satu hari? }\end{array}$ & :............. & & & \\
\hline
\end{tabular}




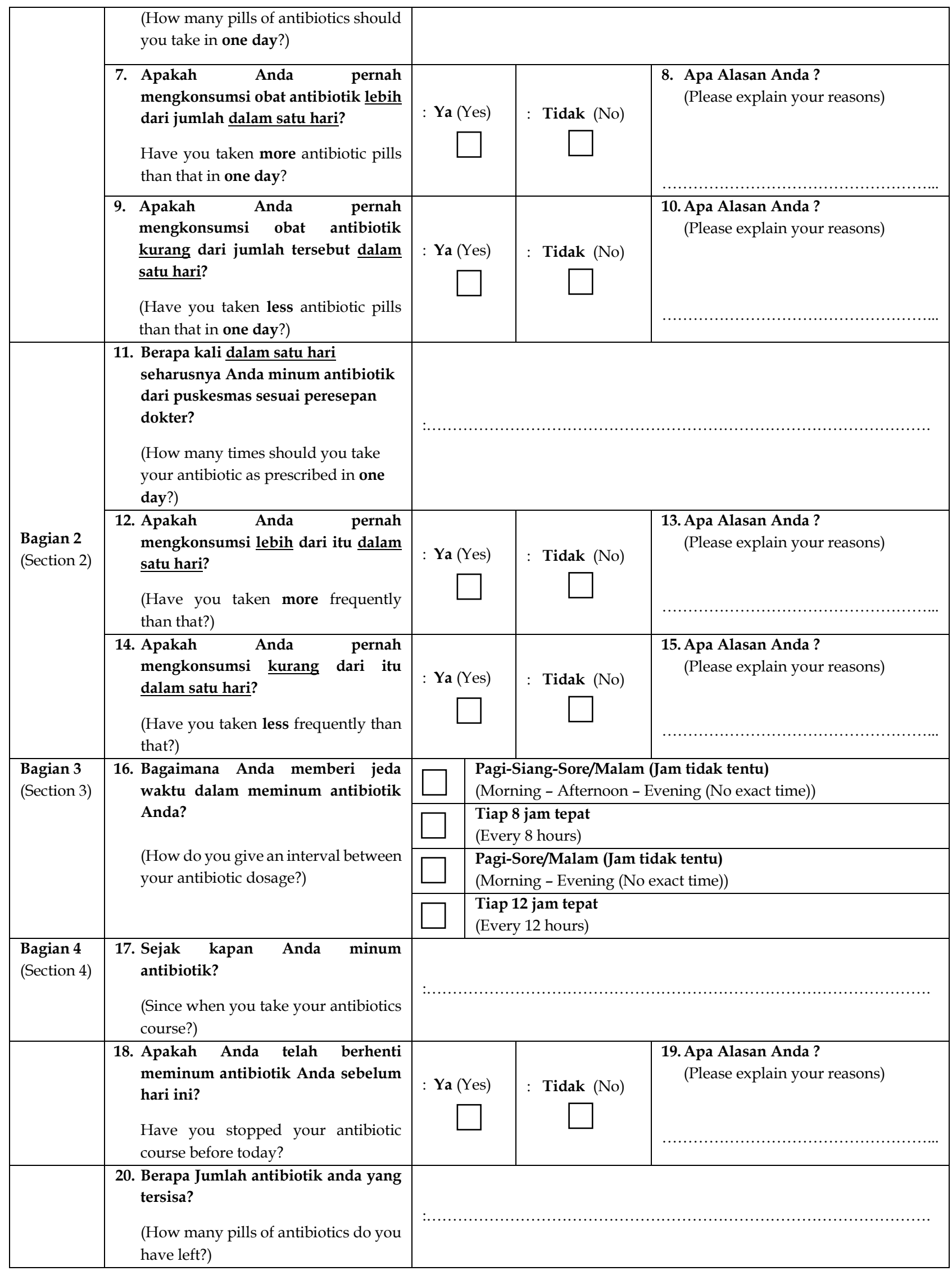

To our knowledge, there had no reported studies developing a questionnaire that evaluated oral antibiotics compliance in Indonesia. A study in Lithuania by Kandrotaite et al. (2013) developed a 91-items 
questionnaire adapted from ASK-20, SF-12, and Morisky scale questionnaire to identify the risk of nonadherence antibiotics treatments. It was said that the developed questionnaire covered the identification of the fivedimension adherence model developed by World Health Organization (Kandrotaite et al., 2013). However, although the developed questionnaire had been discussed with nine professionals, it had not been validated yet. Besides, one of the studies in Indonesia that used questionnaires to measure antibiotics compliance was the study by Muljabar and Supadmi (2014) that used 8-items MMAS. However, the study did not validate the questionnaire directly to patients receiving antibiotics. Therefore, the questionnaire developed in this study could be tested for construct validity and reliability tests. Construct validity is the degree to which an instrument measures the trait or theoretical construct intended to measure, while reliability test is the extent to which a questionnaire produces consistent results on repeated trials (Boateng et al., 2018; Kimberlin \& Winterstein, 2008). Construct validity for the 20-KAO questionnaire is essentials to measure how well the targeted respondents give answers as the questionnaire aims to measure, while the reliability test is useful to assess whether the questionnaire will give consistency in results. Future construct validity and reliability test for 20-KAO questionnaire can be done through distribution to the patient's prescribed antibiotics and then analyze their responses.

\section{CONCLUSION}

The 20-KAO questionnaire was found to have excellent content validity based on six experts' reviews. Future construct validity and reliability tests for 20-KAO are needed to be conducted to analyze the respond of targeted respondents and the consistency of the questionnaire.

\section{ACKNOWLEDGMENT}

The authors would like to thank The Ministry of Education and Culture Republic of Indonesia for the funding support in the scheme of Penelitian Dosen Pemula 2020, the community health centers of Surabaya for the permission, the experts who participated in this study, also Akademi Farmasi Surabaya for the approvals.

\section{REFERENCES}

Boateng, G.O., Neilandsm T.B., Fongillo, E.A., MelgarQuiñonez, H.R., \& Young, S.L. (2018). Best Practices for Developing and Validating Scales for Health, Social, and Behavioral Research: A Primer. Frontiers in Public Health, 6, 149. doi:10.3389/fpubh.2018.00149

Bolarinwa, O.A. (2015). Principles and methods of validity and reliability testing of questionnaires used in social and health science researches. Nigerian Postgraduate Medical Journal, 22(4), 195201. doi:10.4103/1117-1936.173959

Duong, M., Piroth, L., Grappin, M., Forte, F., Peytavin, G., Buisson, M., Chavanet, P., \& Portier, H. (2001). Evaluation of the Patient Medication Adherence Questionnaire as a tool for selfreported adherence assessment in HIVinfected patients on antiretroviral regimens. HIV Clinical Trials, 2(2), 128-135. doi:10.1310/M3JR-G390-LXCM-F62G

Economou, V. \& Gousia, P. (2015). Agriculture and food animals as a source of antimicrobial-resistant bacteria. Infection and Drug Resistance, 8, 49-61. doi:10.2147/IDR.S55778

Fair, R.J. \& Tor, Y. (2014). Antibiotics and Bacterial Resistance in the 21st Century. Perspective in Medicinal Chemistry, 6, 25-64. doi:10.4137/PMC.S14459

Jin, J., Sklar, G.E., Oh, V.M.S., \& Li, S.C. (2008). Factors affecting therapeutic compliance: A review from the patient's perspective. Therapeutics and Clinical Risk Management, 4(1), 269-286. doi:10.2147/tcrm.s1458

Kandrotaite, K., Smigelskas, K., Janusauskiene, D., Jievaltas, M., Maciulaitis, R., \& Briedis, V. (2013). Development of a short questionnaire 
to identify the risk of nonadherence to antibiotic treatment. Current Medical Research and Opinion, 29(11), 1555-1563. doi:10.1185/03007995.2013.835255

Kimberlin, C.L. \& Winterstein, A.G. (2008). Validity and reliability of measurement instruments used in research. American Journal of Health-System Pharmacy, 65(23), 2276-2284. doi:10.2146/ajhp070364

Klein, E.Y., Boeckel, T.P.V., Martinez, E.M., Pant, S., Gandra, S., Levin, S.A., Goossens, H., \& Laxminarayan, R. (2018). Global increase and geographic convergence in antibiotic consumption between 2000 and 2015. Proceedings of the National Academy of Sciences of the United States of America, 115(15), E3463E3470. doi:10.1073/pnas.1717295115

Lam, W.Y. \& Fresco, O. (2015). Medication Adherence Measures: An Overview. BioMed Research International, 2015, 217047. doi:10.1155/2015/217047

Llor, C., Hernández, S., Bayona, C., Moragas, A., Sierra, N., Hernández, M., \& Miravitlles, M. (2013). A study of adherence to antibiotic treatment in ambulatory respiratory infections. International Journal of Infectious Diseases, 17(3), e168-172. doi:10.1016/j.ijid.2012.09.012

Martinez, J.L. (2017). Effect of antibiotics on bacterial populations: a multi-hierachical selection process. F1000Research, 6, 51. doi:10.12688/f1000research.9685.1

Moradi, M., Hamedi-Shahraki, S., Rezayee, M., \& Verdi, M. (2013). Compliance with antimicrobial therapy: Evaluating the related factors. Journal of Pharmaceutical Care, 1(2), 60-64.

Morisky, D.E., Green, L.W., \& Levine, D.M. (1986). Concurrent and predictive validity of a selfreported measure of medication adherence. Medical Care, 24(1), 67-74. doi:10.1097/00005650-198601000-00007

Muljabar, S.M. \& Supadmi, W. (2014). Pengaruh Pemberian Informasi Obat terhadap Tingkat Kepatuhan Penggunaan Antibiotika pada Pasien ISPA di Puskesmas Kotagede I Yogyakarta. Pharmaciana: Jurnal Kefarmasian, 4(2), 143-149. doi:10.12928/pharmaciana.v4i2.1571
Polit, D.F. \& Beck, C.T. (2006). The content validity index: are you sure you know what's being reported? Critique and recommendations. Research in Nursing and Health, 29(5), 489-497. doi:10.1002/nur.20147

Ponto, J. (2015). Understanding and Evaluating Survey Research. Journal of the Advanced Practitioner in Oncology, 6(2), 168-171.

Setia, M.S. (2017). Methodology Series Module 9: Designing Questionnaires and Clinical Record Forms - Part II. Indian Journal of Dermatology, 62(3), 258-261. doi:10.4103/ijd.IJD_200_17

Thompson, K., Kulkarni, J., \& Sergejew, A.A. (2000). Reliability and validity of a new Medication Adherence Rating Scale (MARS) for the psychoses. Schizophrenia Research, 42(3), 241247. doi:10.1016/s0920-9964(99)00130-9

Treibich, C. \& Ventelou, B. (2017). Validation of a shortform questionnaire to check patients' adherence to antibiotic treatments in an outpatient setting. European Journal of Public Health, 27(6), 978-980. doi:10.1093/eurpub/ckx146

Williams, A.B., Amico, K.R., Bova, C., \& Womack, J.A. (2013). A proposal for quality standards for measuring medication adherence in research. AIDS and Behavior, 17(1), 284-297. doi:10.1007/s10461-012-0172-7 